\title{
An evaluation of the safety and efficacy of bimatoprost for eyelash growth in pediatric subjects
}

This article was published in the following Dove Press journal:

Clinical Ophthalmology

10 March 2016

Number of times this article has been viewed

\author{
Mark Borchert' \\ Suzanne Bruce ${ }^{2}$ \\ David Wirta ${ }^{3}$ \\ Steven G Yoelin ${ }^{4}$ \\ Sungwook Lee ${ }^{5}$ \\ Cheri $\mathrm{Mao}^{5}$ \\ Amanda VanDenburgh ${ }^{5}$ \\ 'Children's Hospital Los Angeles, \\ Los Angeles, CA, USA; ${ }^{2}$ Suzanne Bruce \\ and Associates, PA, Houston, TX, USA; \\ ${ }^{3}$ David Wirta and Associates, Newport \\ Beach, CA, USA; ${ }^{4}$ Medical Associates \\ Inc., Newport Beach, CA, USA; \\ ${ }^{5}$ Allergan plc, Irvine, CA, USA
}

Correspondence: Mark Borchert Children's Hospital Los Angeles, 4650 Sunset Boulevard, Los Angeles, CA 90027, USA

$\mathrm{Tel}+\mathrm{I} 32336 \mid 2347$

Fax +l 3233613524

Email mborchert@chla.usc.edu
Purpose: Evaluate the safety and effectiveness of bimatoprost $0.03 \%$ for treatment of eyelash hypotrichosis in a pediatric population.

Patients and methods: This multicenter, randomized, double-masked, parallel-group study was conducted at seven sites in the US and Brazil. Subjects with eyelash hypotrichosis caused by chemotherapy or alopecia areata (aged 5-17 years) or healthy adolescents aged 15-17 years were enrolled $(\mathrm{N}=71)$. Subjects applied bimatoprost $0.03 \%$ or vehicle to upper eyelid margins once nightly for 4 months and were followed for 1 month post-treatment. Eyelash prominence was assessed using the validated 4-grade Global Eyelash Assessment scale with photonumeric guide. Changes in eyelash length, thickness, and darkness were measured by digital image analysis. Safety was assessed by adverse events and ophthalmic observations.

Results: Eyelash prominence improved in a significantly greater proportion of subjects treated with bimatoprost compared with vehicle at month 4 (70.8\% versus $26.1 \% ; P<0.001)$. This benefit was sustained at month 5 post-treatment assessment. Digital image analysis measures were significantly improved with bimatoprost. Significant treatment benefits with bimatoprost versus vehicle were evident among the healthy adolescents but not in the postchemotherapy or alopecia areata subgroups. The safety profile of bimatoprost was consistent with previous studies in adults.

Conclusion: Bimatoprost was safe and well tolerated in pediatric subjects with eyelash hypotrichosis. In this study with limited sample size, subgroup analyses showed that treatment was effective in healthy adolescents with no concurrent contributing medical condition, but not in those with eyelash hypotrichosis due to chemotherapy or alopecia areata.

Keywords: adolescent, child, hypotrichosis, Latisse

\section{Introduction}

Some individuals experience reduced eyelash growth, known as eyelash hypotrichosis, that may be attributed to multiple factors, including aging, heredity, physical trauma, alopecia areata, and chemotherapy. ${ }^{1}$ Alopecia areata, characterized by periods of hair loss and regrowth, typically affects the scalp but may affect eyelashes and eyebrows as well. ${ }^{2}$ It is attributable to autoimmune damage to the hair bulb of anagenic hairs. Cytotoxic chemotherapy agents also induce transient hair loss, in some cases affecting the eyelashes, as they target the rapidly proliferating keratinocytes of anagen hair follicles. Hair regrowth after chemotherapy may be altered ${ }^{3}$ and recover slowly upon the hair follicle entering a new growth cycle from the dormant anagen-arrest state caused by chemotherapy. Natural eyelash regrowth during remittance of alopecia areata and following chemotherapy is not well understood. While treatment is not necessary, it may address the psychological impact of hair loss. 
Although products such as mascara lengthen, thicken, and darken existing eyelashes, they cannot enhance missing lashes and are less effective when eyelashes are sparse. ${ }^{4}$ Options such as eyelash extensions are associated with an increased risk of corneal and eyelid infection and with temporary or permanent loss of existing eyelashes. ${ }^{5}$ Bimatoprost, a synthetic prostaglandin analog, stimulates resting hair follicles to enter a new growth phase and may be useful in accelerating eyelash regrowth in chemotherapy- or alopecia areata-induced hypotrichosis. ${ }^{6}$

Bimatoprost was originally approved in 2001 for treatment of open-angle glaucoma and ocular hypertension. Eyelash growth was observed as an adverse event (AE) in clinical trials, leading to evaluations for treatment of eyelash hypotrichosis. ${ }^{7-10}$ Once-nightly application of bimatoprost $0.03 \%$ to the upper eyelid margin increased eyelash length, thickness, and darkness compared with vehicle in adults with idiopathic and chemotherapy-induced eyelash hypotrichosis. ${ }^{11,12}$ Bimatoprost ophthalmic solution $0.03 \%$ was approved for treatment of eyelash hypotrichosis in adults in 2008. ${ }^{13}$ This study fulfills a Food and Drug Administration postmarketing commitment to provide data on the safety of bimatoprost $0.03 \%$ for the treatment of eyelash hypotrichosis in a pediatric population. This report describes the effects of bimatoprost in children aged 5-17 years with chemotherapyor alopecia areata-induced hypotrichosis, and in healthy adolescents aged 15-17 years with no concurrent contributing medical condition.

\section{Methods}

This multicenter, randomized, double-masked, parallelgroup study was conducted between June 2010 and November 2012 at six sites in the US and one site in Brazil. Clinical trials identifier: NCT01023841. The protocol was approved by an institutional review board or independent ethics committee at each site, and the study was conducted in compliance with Good Clinical Practice guidelines. Consent from a parent or legal guardian and assent from the minor subject were obtained before enrollment.
The study had a 5-month duration, comprising a 4-month treatment period and a 1-month post-treatment follow-up period. At baseline, eligible subjects were stratified by age group (5-11 versus $12-17$ years) and randomly assigned $2: 1$ to receive either bimatoprost $0.03 \%$ or vehicle. Subjects and/or their parents/legal guardians were instructed to apply one drop of study treatment to a sterile, single use per eyelid applicator, and then brush it along the subject's upper eyelid margin once daily in the evening for 4 months. A second drop applied to a second applicator was used to treat the contralateral eyelid margin. During the entire study period, subjects, investigators, and investigational staff remained masked to treatment assignment. All treatments were identical in appearance and supplied in identical bottles.

\section{Subjects}

Initially, children aged 5-17 years with chemotherapyinduced eyelash hypotrichosis were enrolled. However, after difficulty with enrollment, the study population was broadened to include children aged 5-17 years with alopecia areata-induced eyelash hypotrichosis and healthy adolescents aged 15-17 years with no concurrent contributing medical condition (Table 1). All eligible subjects required a Global Eyelash Assessment (GEA) score of 1, 2, or 3; a best-corrected visual acuity score equivalent to a Snellen acuity of 20/100 or better; and intraocular pressure (IOP) $\leq 20 \mathrm{mmHg}$. Females of childbearing potential had to have a negative urine pregnancy test before receiving study treatment. Subjects with chemotherapy-induced eyelash hypotrichosis also had to have completed intensive chemotherapy at least 4 weeks before study baseline; have a low risk of cancer relapse for at least 6 months at the time of study entry; and have chemotherapyrelated AEs resolved or adequately managed (except for hair loss). Subjects on maintenance chemotherapy were eligible. Subjects were excluded if they presented with gross asymmetry of eyelash prominence between the left and right eyes, uncontrolled systemic disease (other than cancer for postchemotherapy subjects), known or suspected trichotillomania, or known ocular abnormality or disease.

Table I Sample size of each etiology by age group

\begin{tabular}{|c|c|c|c|c|c|c|c|c|}
\hline \multirow[t]{2}{*}{$\begin{array}{l}\text { Age group, } \\
\text { years }\end{array}$} & \multicolumn{2}{|c|}{$\begin{array}{l}\text { Overall } \\
(\mathrm{N}=7 \mathrm{I})\end{array}$} & \multicolumn{2}{|c|}{$\begin{array}{l}\text { Healthy adolescent } \\
(n=40)\end{array}$} & \multicolumn{2}{|c|}{$\begin{array}{l}\text { Postchemotherapy } \\
(n=16)\end{array}$} & \multicolumn{2}{|c|}{$\begin{array}{l}\text { Alopecia areata } \\
(n=15)\end{array}$} \\
\hline & BIM & Vehicle & BIM & Vehicle & BIM & Vehicle & BIM & Vehicle \\
\hline $5-11$ & 6 & 3 & 0 & 0 & 3 & I & 3 & 2 \\
\hline $12-14$ & 6 & 4 & 0 & 0 & 3 & I & 3 & 3 \\
\hline $15-17$ & 36 & 16 & 26 & 14 & 7 & I & 3 & I \\
\hline
\end{tabular}

Abbreviation: BIM, bimatoprost. 


\section{Efficacy assessments}

Subjects were evaluated at screening, on day 1 , and at months $1,2,3,4$, and 5. Efficacy was evaluated using physician-rated GEA score of prominence, digital image analysis (DIA) of photographs to assess the individual components of eyelash prominence (length, thickness, and darkness), and efficacy from the subject's point of view using the validated 3-item Eyelash Satisfaction Questionnaire (ESQ-3). Eyelash prominence was assessed by investigators using the validated 4-point GEA scale with photonumeric guide (1=minimal; 2=moderate; $3=$ marked; $4=$ =very marked). Digital eyelash photographs were taken at baseline and at each post-treatment visit using standardized equipment. DIA was used to assess upper eyelash length in millimeters, eyelash thickness in square millimeters, and eyelash darkness in intensity units (IU). Subjects aged 12-17 years completed the validated ESQ-3, which assesses subject satisfaction with the objective attributes of eyelashes (length, fullness, and overall satisfaction); responses were collected using a 5-point Likert-type scale (very satisfied to very unsatisfied). The ESQ-3 was administered only to subjects aged $12-17$ years because the questionnaire was expected to be understandable and relevant only to these older subjects.

\section{Safety assessments}

AEs were monitored throughout the study and were recorded by investigators at the study entry and at each postbaseline visit. Physical examinations with vital signs were performed at screening and at each postbaseline office visit. Ophthalmic examinations of IOP, best-corrected visual acuity, biomicroscopy, and iris color were conducted for each subject at screening/baseline and at months 1 and 4 ; dilated ophthalmoscopy was performed at screening/baseline and at month 4 .

\section{Data analysis and statistics}

Sample size calculations were determined empirically to assess the safety profile of bimatoprost $0.03 \%$ in a pediatric population with different hypotrichosis etiologies. Although this study was not powered for efficacy, efficacy variables were nonetheless evaluated in the intent-to-treat population, comprising all randomized subjects; safety parameters were evaluated in all subjects who received at least one dose of study treatment (safety population). Comparisons of responder rates, defined as the proportion of subjects who had at least a 1-grade improvement from baseline in GEA scores at month 4 , between the bimatoprost and vehicle groups were performed using the Cochran-Mantel-Haenszel method, stratified by age group. A last observation carried forward method was used to impute missing values from months 1 to 4 . For assessment of changes from baseline in DIA measures of eyelash length, thickness, and darkness, increased length and thickness were shown by a positive change from baseline, whereas increased darkness presented as a negative change in IU from baseline. Comparisons of DIA measures between treatment groups were made using the van Elteren test. ESQ-3 score responses were also dichotomized into two groups (satisfied and not satisfied/ neutral). Between-group comparisons of ESQ-3 responses were performed using the Wilcoxon rank-sum test, and of dichotomized ESQ-3 responses using Pearson's chi-square or Fisher's exact test. AEs were summarized by frequency distribution, with between-group comparisons made using Fisher's exact test.

\section{Results}

In total, 71 subjects with eyelash hypotrichosis were randomized to receive bimatoprost $(n=48)$ or vehicle $(n=23)$. One bimatoprost-treated subject discontinued due to an exacerbation of facial eczema, considered not related to study treatment. The treatment groups were similar with regard to baseline characteristics (Table 2).

In the postchemotherapy subgroup, cancer diagnoses included sarcomas $(n=8)$, hematologic malignancies $(n=7)$, and Askin's tumor $(\mathrm{n}=1)$. This subgroup completed their most recent chemotherapy within a median of 47.5 (range, 30-765) days before study treatment; median was 50 days for 13 subjects receiving bimatoprost and 45 days for three subjects receiving vehicle. Most $(\mathrm{n}=13)$ received a cytotoxic combination chemotherapy regimen; the remainder $(n=3)$ received single-agent methotrexate or etoposide.

\section{Efficacy}

In the total population, a significantly greater proportion of bimatoprost-treated subjects versus vehicle demonstrated a 1 -grade or greater increase from baseline in GEA score at month $4(P<0.001)$, which was evident by month 3 and sustained at month 5 (Figure 1). When analyzed by hypotrichosis etiology, significant between-treatment differences favored bimatoprost over vehicle in healthy adolescents at month 4 $(P<0.001)$, despite the relatively high median GEA score of 2.9 at baseline for these groups, regardless of treatment. Similarly, significant improvements in eyelash thickness were seen at months $1,3,4$, and 5 in the entire cohort and in healthy adolescents. Improvements in eyelash length were significantly greater with bimatoprost versus vehicle at months 3,4 , and 5 in healthy adolescents, but for the entire 
Table 2 Baseline characteristics

\begin{tabular}{|c|c|c|c|}
\hline Characteristic & $\begin{array}{l}\text { Bimatoprost } \\
(n=48)\end{array}$ & $\begin{array}{l}\text { Vehicle } \\
(n=23)\end{array}$ & $\begin{array}{l}\text { Total } \\
(N=7 \text { I) }\end{array}$ \\
\hline Age, mean (range), years & $14.5(5-17)$ & $14.6(8-17)$ & $14.5(5-17)$ \\
\hline $5-11$ years, n (\%) & $6(12.5)$ & $3(13.0)$ & $9(12.7)$ \\
\hline 12-17 years, n (\%) & $42(87.5)$ & $20(87.0)$ & $62(87.3)$ \\
\hline Female, n (\%) & $37(77.1)$ & $16(69.6)$ & $53(74.6)$ \\
\hline \multicolumn{4}{|l|}{ Race, n (\%) } \\
\hline White & $31(64.6)$ & $15(65.2)$ & $46(64.8)$ \\
\hline Black & $0(0)$ & $3(13.0)$ & $3(4.2)$ \\
\hline Asian & $2(4.2)$ & $0(0)$ & $2(2.8)$ \\
\hline Hispanic & $12(25.0)$ & $5(21.7)$ & $17(23.9)$ \\
\hline Other & $3(6.3)$ & $0(0)$ & $3(4.2)$ \\
\hline \multicolumn{4}{|l|}{ Iris color, ${ }^{a}$ n (\%) } \\
\hline Dark & $34(70.8)$ & II (47.8) & $45(63.4)$ \\
\hline Light & $14(29.2)$ & $12(52.2)$ & $26(36.6)$ \\
\hline \multicolumn{4}{|l|}{ GEA score, $\mathrm{n}(\%)$} \\
\hline I (none/minimal) & $10(20.8)$ & $9(39.1)$ & $19(26.8)$ \\
\hline 2 (moderate) & II (22.9) & I (4.3) & $12(16.9)$ \\
\hline 3 (marked) & $27(56.3)$ & $13(56.5)$ & $40(56.3)$ \\
\hline 4 (very marked) & $0(0)$ & $0(0)$ & $0(0)$ \\
\hline \multicolumn{4}{|l|}{ Etiology, n (\%) } \\
\hline Postchemotherapy ${ }^{\mathrm{b}}$ & I3 (27.I) & $3(13.0)$ & $16(22.5)$ \\
\hline Alopecia areata & $9(18.8)$ & $6(26.1)$ & $15(21.1)$ \\
\hline Healthy adolescent & $26(54.2)$ & $14(60.9)$ & $40(56.3)$ \\
\hline
\end{tabular}

Notes: a Dark iris color included brown, dark brown, and black; light iris color included blue, blue-gray, blue/gray-brown, green, green-brown, gray, and hazel. ${ }^{b} \mathrm{C} a n c e r$ types in the bimatoprost group included acute lymphoblastic leukemia $(n=1)$, alveolar rhabdomyosarcoma $(n=l)$, Askin's tumor $(n=I)$, Ewing's sarcoma $(n=I)$, Hodgkin lymphoma $(n=2)$, mediastinal $B$ cell lymphoma $(n=1)$, non-Hodgkin lymphoma $(n=1)$, osteosarcoma $(n=2)$, pelvic osteosarcoma $(n=l)$, tibial osteosarcoma $(n=l)$, and osteosarcoma of the mandible $(n=1)$. Cancer types in the vehicle group included acute lymphoblastic leukemia $(n=I)$, Hodgkin lymphoma $(n=I)$, and rhabdomyosarcoma $(n=I)$. Abbreviation: GEA, Global Eyelash Assessment.

cohort, between-group differences neared statistical significance at months 3 and 4 , and reached statistical significance at month 5 .

Among those with chemotherapy-induced eyelash hypotrichosis, most receiving bimatoprost and all receiving vehicle had $\geq 1$-grade increases from baseline in GEA scores at month 4. Overall, 11 of 13 postchemotherapy subjects achieved $\geq 1$-grade improvement from baseline in GEA scores, as did all three vehicle-treated postchemotherapy subjects. However, the mean baseline score was nearly 1 grade higher for subjects receiving bimatoprost versus vehicle (1.9 versus 1.0 , respectively). Between-group comparisons at month 4 were not significant in subjects with alopecia areata (Figure 1). Again, the mean baseline score was higher for subjects receiving bimatoprost versus vehicle (1.4 versus 1.0, respectively). Mean change from baseline in GEA of 1 grade at month 4 in a healthy adolescent and a postchemotherapy subject representative of these subpopulations is shown in Figure 2.

The mean increase from baseline in upper eyelash length in the total population was greater with bimatoprost versus vehicle; the difference approached statistical significance at month 4 ( 1.12 versus $0.67 \mathrm{~mm} ; P=0.055)$ and reached significance at month 5 (1.21 versus $0.79 \mathrm{~mm} ; P=0.017)$ (Figure S1). In the healthy adolescents, between-group comparison favored bimatoprost over vehicle for months 3-5; mean increase from baseline at month 4 was $0.41 \mathrm{~mm}$ with bimatoprost versus $-0.31 \mathrm{~mm}$ with vehicle $(P=0.004)$. Eyelash length increased with both treatments in subjects with chemotherapy- or alopecia areata-induced eyelash hypotrichosis, but between-group comparisons were not significant. At baseline, however, mean eyelash length was greater with bimatoprost versus vehicle for both subgroups, although between-group comparisons were not significant.

Significant improvements in upper eyelash thickness were evident at months 1, 3, 4, and 5 in the total population following treatment with bimatoprost versus vehicle. At month 4 , the mean increase from baseline with bimatoprost was 0.41 versus $0.05 \mathrm{~mm}^{2}$ with vehicle in the total study cohort $(P=0.002$; Figure $\mathrm{S} 2)$. In the healthy adolescents, significant improvements in upper eyelash thickness were also evident at months 1, 3, 4, and 5; mean increases from baseline at month 4 with bimatoprost were 0.23 versus $-0.20 \mathrm{~mm}^{2}$ with vehicle $(P<0.001)$. No significant improvements in upper eyelash thickness with bimatoprost versus vehicle were noted in the postchemotherapy or alopecia areata subgroups.

Upper eyelash darkness improved significantly with bimatoprost versus vehicle at months 3 and 4 in the total population $(P<0.05$; Figure S3). At month 4 , the mean change from baseline was -15.34 IU with bimatoprost versus $-5.47 \mathrm{IU}$ with vehicle $(P=0.019)$. No significant differences in eyelash darkness were observed between the subgroups. However, trends toward improvement favoring bimatoprost versus vehicle were noted in the healthy adolescent $(P=0.089$, month 4$)$ and alopecia areata $(P=0.083$, months 2-5) subgroups.

The ESQ-3 was completed by subjects aged $12-17$ years ( $n=42$, bimatoprost group; $n=20$, vehicle group). Mean scores for satisfaction on each of the three items improved from baseline in both treatment groups (Figure 3). However, betweengroup differences did not reach statistical significance. At month 4 , a numerically greater proportion of subjects receiving bimatoprost versus vehicle reported being satisfied or very satisfied with eyelash length, eyelash thickness/fullness, and overall satisfaction with their eyelashes.

\section{Safety}

Seventeen of 48 pediatric subjects (35.4\%) in the total population who received bimatoprost and 10 of 23 subjects (43.5\%) who received vehicle reported at least one $\mathrm{AE}$ during the 
A

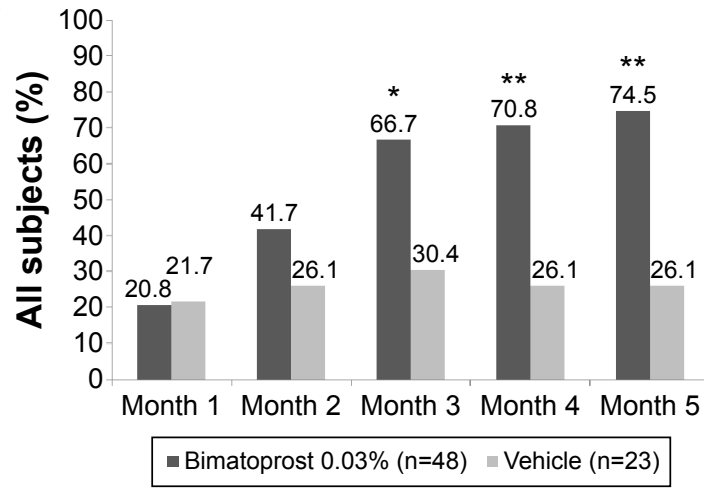

C

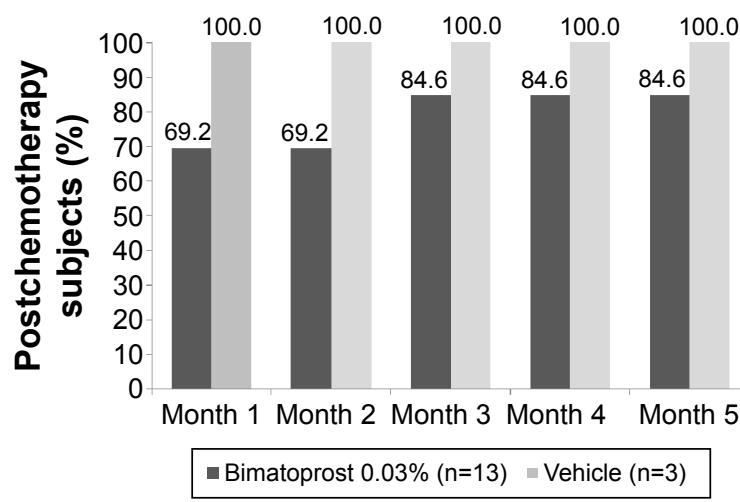

B

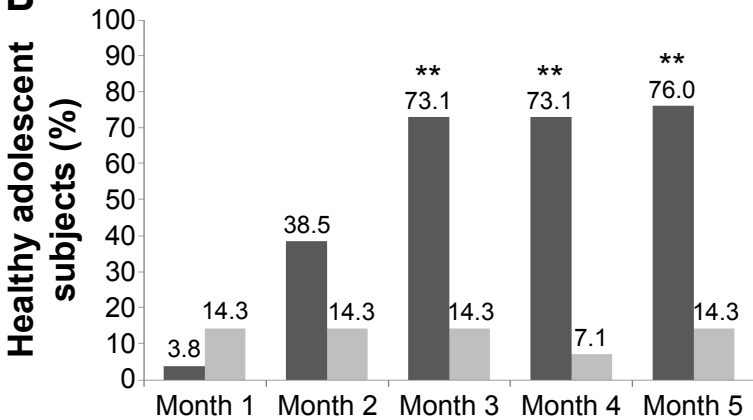

- Bimatoprost $0.03 \%(n=26) \quad$ Vehicle $(n=14)$

D

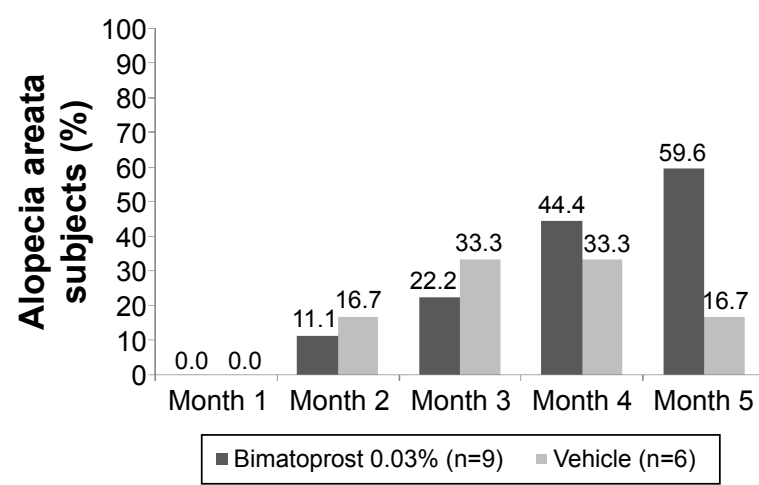

Figure I Percentage of pediatric subjects with at least a I-grade improvement from baseline in Global Eyelash Assessment scores.

Notes: (A) Overall population. (B) Healthy adolescents. (C) Postchemotherapy pediatric subjects. (D) Pediatric subjects with alopecia areata. Study treatment was applied once nightly for 4 months. Subjects did not use study treatment between months 4 and 5 . $* P=0.004$ versus vehicle. $* * P<0.00$ I versus vehicle.

study. All AEs were mild or moderate in intensity. No severe or serious AEs were reported, nor did any deaths occur. No clinically meaningful differences in AE rates by etiology of eyelash hypotrichosis were noted. In the bimatoprost and vehicle groups, AEs occurred in 7/26 (26.9\%) and 6/14 (42.9\%) healthy adolescents, respectively; in 6/13 (46.2\%) and $2 / 3(66.7 \%)$ postchemotherapy subjects, respectively; and in 4/9 (44.4\%) and 2/6 (33.3\%) subjects with alopecia areata.
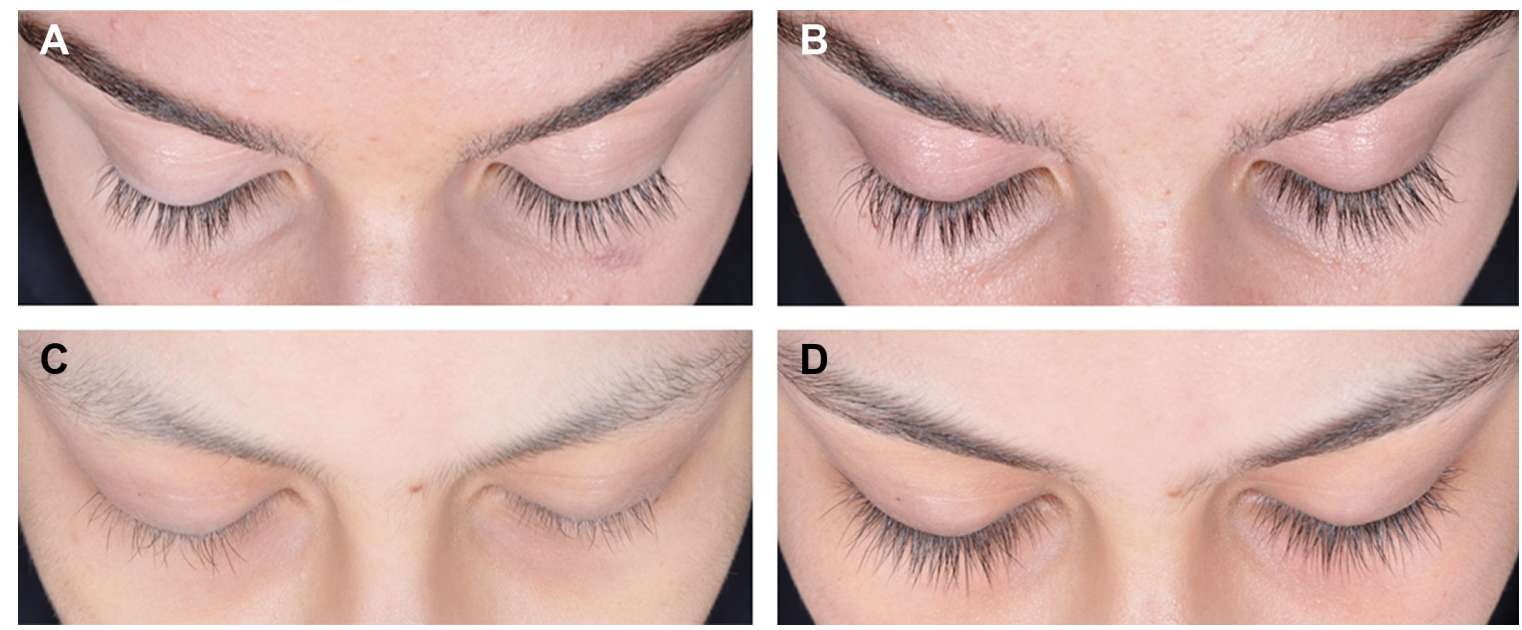

Figure 2 Examples of response to bimatoprost treatment with a I-grade improvement in GEA score from baseline to month 4.

Notes: In a healthy subject aged 17 years, GEA score at baseline was 3 (A) and improved to GEA score of 4 at month 4 (B). In a postchemotherapy subject aged I 3 years, baseline GEA score of 2 (C) improved to GEA score of 3 at month 4 (D).

Abbreviation: GEA, Global Eyelash Assessment. 

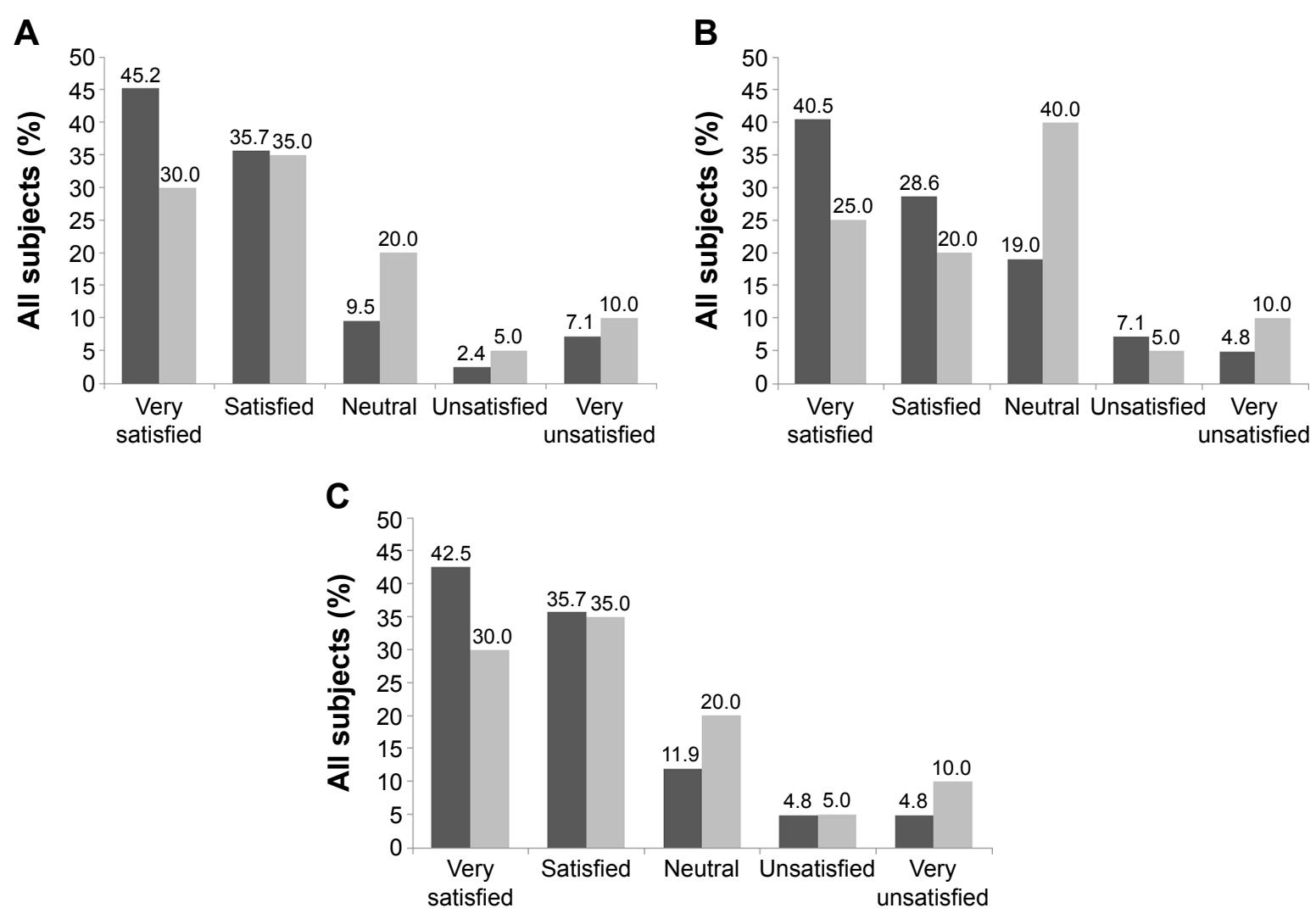

Bimatoprost $0.03 \%(n=42) \backsim$ Vehicle $(n=20)$

Figure 3 Responses to the ESQ-3 at month 4 for (A) satisfaction with eyelash length, (B) satisfaction with eyelash fullness/thickness, and (C) overall eyelash satisfaction for the overall pediatric population aged $12-17$ years.

Notes: Study treatment was applied once nightly for 4 months; subjects did not use study treatment between months 4 and 5 .

Abbreviation: ESQ-3, 3-item Eyelash Satisfaction Questionnaire.

The most frequently reported AEs in subjects receiving bimatoprost are given in Table 3. No iris or skin hyperpigmentation was reported. Treatment-related AEs occurred in eight subjects $(16.7 \%)$ receiving bimatoprost (four postchemotherapy subjects [30.8\%], three healthy adolescents [11.5\%], and one alopecia areata subject [11.1\%]). No treatment-related AEs occurred in the vehicle group. Most treatment-related AEs in the bimatoprost group were related to topical events local to the application site; none occurred in more than one subject by etiology. Besides the aforementioned discontinuation due to exacerbation of facial eczema in a healthy adolescent (considered unrelated to study treatment), there were no other study discontinuations.

Ophthalmic examinations detected no statistically significant or clinically meaningful differences between bimatoprost and vehicle groups in mean change from baseline in IOP at months 1 or 4 in the overall safety population; peak mean change from baseline was -1.12 versus $-0.87 \mathrm{mmHg}$, respectively, observed at month $1(P=0.658)$. Similarly, no statistically significant or clinically meaningful differences in this parameter occurred in the alopecia areata or postchemotherapy subgroups. A statistically significantly greater mean

Table 3 Adverse events reported in more than one subject in the overall population treated with bimatoprost

\begin{tabular}{|c|c|c|c|c|}
\hline \multirow[t]{2}{*}{ Adverse event, n (\%) } & \multicolumn{4}{|c|}{ Bimatoprost $0.03 \%^{\mathrm{a}}$} \\
\hline & Overall $(\mathrm{N}=48)$ & Healthy adolescent $(n=26)$ & Postchemotherapy $(n=13)$ & Alopecia areata $(n=9)$ \\
\hline Conjunctival hyperemia & $2(4.2)$ & $\mathrm{I}(3.8)$ & 0 & I (II.I) \\
\hline Conjunctivitis & $2(4.2)$ & 0 & I (7.7) & I (II.I) \\
\hline Eczema & $2(4.2)$ & I (3.8) & 0 & I (II.I) \\
\hline Erythema of eyelid & $2(4.2)$ & I (3.8) & 0 & I (II.I) \\
\hline Nasopharyngitis & $2(4.2)$ & 0 & $2(15.4)$ & 0 \\
\hline Sinusitis & $2(4.2)$ & 0 & $2(15.4)$ & 0 \\
\hline
\end{tabular}

Note: ${ }^{a}$ No adverse events were reported in $>$ I subject in the vehicle group of the overall population $(n=23)$. 
decrease from baseline IOP was reported for healthy adolescents receiving bimatoprost versus vehicle at month 4 (mean change, -1.29 versus $-0.23 \mathrm{mmHg}$, respectively; $P=0.045$ ). However, these changes were not clinically meaningful and are well within the diurnal phasic variations in IOP. ${ }^{14}$

Two subjects had an IOP $\leq 6 \mathrm{mmHg}$ in either eye at any time during the study. One alopecia areata subject in the bimatoprost group had declines in IOP of $43 \%$ in the right eye $(10.5 \mathrm{mmHg}$ at baseline; $6 \mathrm{mmHg}$ at month 4$)$ and $33 \%$ in the left eye $(9 \mathrm{mmHg}$ at baseline; $6 \mathrm{mmHg}$ at month 4). No subsequent follow-up was reported. In the other, a healthy adolescent who received vehicle, IOP declined by $57 \%$ in the right eye $(15 \mathrm{mmHg}$ at baseline; $6.5 \mathrm{mmHg}$ at month 1$)$ and $52 \%$ in the left eye $(12.5 \mathrm{mmHg}$ at baseline; $6 \mathrm{mmHg}$ at month 1); IOP returned to near baseline in both eyes at month 4 . These changes in IOP were not considered clinically meaningful, and AEs were not reported for either subject.

Visual acuity (change defined as \pm 2 lines) remained unchanged in all subjects receiving vehicle and in most subjects $(>93.6 \%)$ receiving bimatoprost from baseline to month 1 or 4 . Biomicroscopy and ophthalmoscopy findings of at least a 1-grade increase in severity from baseline occurred slightly more frequently with bimatoprost $(5 / 48$; $10.4 \%)$ versus vehicle $(2 / 23 ; 8.7 \%)$. One alopecia areata subject in the vehicle group had a 2-grade increase in severity on biomicroscopy and ophthalmoscopy for growth of eyelashes, but the eyelashes were described as still sparse and thin.

\section{Discussion}

This randomized clinical trial demonstrated that bimatoprost $0.03 \%$ applied once nightly to the upper eyelid margins for a 4-month period was safe in subjects aged 5-17 years. Reported AEs were consistent with the known safety profile of bimatoprost $0.03 \%$ established in adults. ${ }^{12}$ Moreover, no clinically meaningful differences in AEs were detected by eyelash hypotrichosis etiology.

Although this study was not powered to evaluate efficacy, bimatoprost improved eyelash prominence, length, and thickness relative to vehicle in the total population and in healthy adolescents. Improvements from baseline in eyelash prominence, length, and thickness were also noted with bimatoprost in the alopecia areata and postchemotherapy subgroups; however, difference compared with the vehicle group did not reach statistical significance. In alopecia areata and postchemotherapy subgroups, mean eyelash length at baseline was greater among bimatoprost-treated versus vehicle-treated subjects. Such baseline score differences across treatment groups may have obscured the treatment benefit of bimatoprost, as there is a greater opportunity for improvement in eyelash prominence with a lower baseline score. It should be noted that mean eyelash length at baseline in the healthy adolescents did not differ between treatment groups.

In contrast to the present findings, postchemotherapy studies in adults have demonstrated eyelash growth enhancements with bimatoprost $0.03 \%$ versus vehicle. ${ }^{11,15,16}$ In 130 adults with chemotherapy-induced eyelash hypotrichosis, bimatoprost restored eyelash growth and prominence more rapidly than natural regrowth in vehicle-control subjects. ${ }^{16}$ Similarly, in a split-face study, significantly increased eyelash length $(P=0.02)$ and thickness $(P=0.04)$ occurred in the treated eyelids of 20 adult postchemotherapy subjects versus the contralateral control eyelids. ${ }^{15}$ In 36 female Japanese subjects with chemotherapy-induced eyelash hypotrichosis, treatment with bimatoprost $0.03 \%$ resulted in significantly longer, thicker, darker, and more prominent eyelashes versus vehicle $(P \leq 0.04) .{ }^{11}$ In this study, most postchemotherapy subjects had received combination cytotoxic chemotherapy regimens. Besides potential differences in the specific chemotherapy drugs used across these studies, it is possible that natural eyelash regrowth after chemotherapy may occur more rapidly in pediatric subjects than in adults. Moreover, while the pediatric subjects completed chemotherapy mostly within 30-120 days before randomization, the mean interval (range) from last treatment to baseline for subjects randomized to the bimatoprost group was double that for those in the vehicle group (112 days [33-765 days] versus 54 days [30-87 days], respectively). Natural eyelash regrowth may have occurred in these subjects prior to bimatoprost treatment, possibly obscuring any effect of bimatoprost on eyelash length, thickness, and darkness.

Previous reports on the efficacy of bimatoprost in the treatment of alopecia areata-induced eyelash hypotrichosis vary considerably, ranging from no growth to complete growth. ${ }^{17-20}$ The present results fall within this wide range: some pediatric subjects had improvements in eyelash prominence and other outcome measures with bimatoprost, although the findings did not differ significantly from those with vehicle. Again, caution must be exercised in interpreting results in this subgroup due to the small sample size. Eyelash growth was not expected to occur in this subpopulation because of the nature of the disease. ${ }^{21}$

The effects of bimatoprost on GEA scores and eyelash length and thickness in the total population were supported largely by effects in the healthy subgroup, adolescents aged 15-17 years. This age group made up $73.2 \%$ of the entire study population. All healthy adolescents in this study 
were aged 15-17 years, whereas the postchemotherapy and alopecia areata cohorts were aged 5-17 years. Statistical comparisons between bimatoprost and vehicle treatment of alopecia areata and postchemotherapy subgroups were limited by the small sample sizes. Because this study was not powered to demonstrate efficacy due to the small population size, any conclusions regarding efficacy should be interpreted with caution.

\section{Conclusion}

These results demonstrate that topical application of bimatoprost $0.03 \%$ to the eyelid margin once nightly is safe and well tolerated in pediatric subjects aged 5-17 years, regardless of the etiology of eyelash hypotrichosis. Bimatoprost was effective for the treatment of idiopathic hypotrichosis in healthy adolescents, but had no significant effect compared with vehicle on hypotrichosis caused by chemotherapy or alopecia areata. Larger clinical trials are needed to draw definitive conclusions on the effectiveness of bimatoprost on eyelash hypotrichosis of varying etiologies in children and adolescents.

\section{Acknowledgments}

The authors thank the subjects, study coordinators, clinical staff, and operations staff who participated in this study. Writing and editorial assistance was provided to the authors by Barry Weichman, $\mathrm{PhD}$, and Kristin E Larsen, PhD, of Peloton Advantage, Parsippany, NJ, USA, and was funded by Allergan plc, Dublin, Ireland. All authors met the ICMJE authorship criteria. Neither honoraria nor other form of payments were made for authorship.

\section{Disclosure}

Dr Borchert received research funding for this study from Allergan plc. Dr Bruce is a consultant for Allergan plc, Lithera, Lumenis, and Ulthera, Inc, and serves as an investigator for AbGenomics, Actavis, Inc., Allergan plc, Anacor Pharmaceuticals, Inc., Braintree Laboratories, Inc., Cipher Pharmaceuticals, Inc., DUSA Pharmaceuticals, Inc., Galderma R\&D Inc., G\&E Herbal Biotechnology Co. Ltd., G\&W Laboratories, Inc., Health Outcomes Solutions, LEO Pharma, Inc., Lithera, Maruho Co. Ltd., Novartis Pharmaceuticals Corp., Obagi Medical Products, Inc., Pfizer, Promius Pharma, LLC, Ranbaxy Laboratories, Ltd., Revance Therapeutics, Inc., Stiefel Laboratories, Inc., Suneva Medical, Inc., Taro Pharmaceutical Industries, Ltd., Tigercat Industries Inc., Tolmar, Inc., and Watson Pharmaceuticals, Inc. Dr Yoelin is a consultant and investigator for Allergan plc. Dr Wirta is a consultant for and has received research grants from Allergan plc. Dr Lee, Ms Mao, and Dr VanDenburgh are employees of Allergan plc. and may own stock or options in that company. This study was sponsored by Allergan plc, Dublin, Ireland. The authors report no other conflicts of interest in this work.

\section{References}

1. Law SK. Bimatoprost in the treatment of eyelash hypotrichosis. Clin Ophthalmol. 2010;4:349-358.

2. Kutner A, Friedman A. Hair loss in the dermatology office: an update on alopecia areata. J Drugs Dermatol. 2013;12:588-593.

3. Paus R, Haslam IS, Sharov AA, Botchkarev VA. Pathobiology of chemotherapy-induced hair loss. Lancet Oncol. 2013;14:e50-e59.

4. Draelos ZD. Special considerations in eye cosmetics. Clin Dermatol. 2001;19:424-430.

5. American Academy of Ophthalmology. American Academy of Ophthalmology warns consumers about the dangers of eyelash extensions [press release]. San Francisco, CA: American Academy of Ophthalmology. Available from: http://www.aao.org/newsroom/news-releases/detail/ american-academy-of-ophthalmology-warnsconsumers-. Accessed December 18, 2015.

6. Cohen JL. Enhancing the growth of natural eyelashes: the mechanism of bimatoprost-induced eyelash growth. Dermatol Surg. 2010;36: 1361-1371.

7. Higginbotham EJ, Schuman JS, Goldberg I, et al. One-year, randomized study comparing bimatoprost and timolol in glaucoma and ocular hypertension. Arch Ophthalmol. 2002;120:1286-1293.

8. Whitcup SM, Cantor LB, VanDenburgh AM, Chen K. A randomised, double masked, multicentre clinical trial comparing bimatoprost and timolol for the treatment of glaucoma and ocular hypertension. $\mathrm{Br} J$ Ophthalmol. 2003;87:57-62.

9. Williams RD, Cohen JS, Gross RL, Liu CC, Safyan E, Batoosingh AL. Long-term efficacy and safety of bimatoprost for intraocular pressure lowering in glaucoma and ocular hypertension: year 4. Br J Ophthalmol. 2008;92:1387-1392.

10. Fagien S. Management of hypotrichosis of the eyelashes: focus on bimatoprost. Clin Cosmet Investig Dermatol. 2010;3:39-48.

11. Harii K, Arase S, Tsuboi R, Weng E, Daniels S, VanDenburgh A. Bimatoprost for eyelash growth in Japanese subjects: two multicenter controlled studies. Aesthetic Plast Surg. 2014;38:451-460.

12. Smith S, Fagien S, Whitcup SM, et al. Eyelash growth in subjects treated with bimatoprost: a multicenter, randomized, double-masked, vehicle-controlled, parallel-group study. J Am Acad Dermatol. 2012;66:801-806.

13. Wirta D, VanDenburgh AM, Weng E, Whitcup SM, Kurstjens S, Beddingfield FC III. Long-term safety evaluation of bimatoprost ophthalmic solution $0.03 \%$ : a pooled analysis of six double-masked, randomized, active-controlled clinical trials. Clin Ophthalmol. 2011;5:759-765.

14. David R, Zangwill L, Briscoe D, Dagan M, Yagev R, Yassur Y. Diurnal intraocular pressure variations: an analysis of 690 diurnal curves. $\mathrm{Br} J$ Ophthalmol. 1992;76:280-283.

15. Morris CL, Stinnett S, Woodward J. The role of bimatoprost eyelash gel in chemotherapy-induced madarosis: an analysis of efficacy and safety. Int J Trichology. 2011;3:84-91.

16. Ahluwalia GS. Safety and efficacy of bimatoprost solution $0.03 \%$ topical application in patients with chemotherapy-induced eyelash loss. J Investig Dermatol Symp Proc. 2013;16:S73-S76.

17. Ochoa BE, Sah D, Wang G, Stamper R, Price VH. Instilled bimatoprost ophthalmic solution in patients with eyelash alopecia areata. $\mathrm{J} \mathrm{Am} \mathrm{Acad}$ Dermatol. 2009;61:530-532.

18. Roseborough I, Lee H, Chwalek J, Stamper RL, Price VH. Lack of efficacy of topical latanoprost and bimatoprost ophthalmic solutions in promoting eyelash growth in patients with alopecia areata. $J$ Am Acad Dermatol. 2009;60:705-706. 
19. Vila TO, Camacho Martinez FM. Bimatoprost in the treatment of eyelash universalis alopecia areata. Int J Trichology. 2010;2:86-88.

20. Zaheri S, Hughes B. Successful use of bimatoprost in the treatment of alopecia of the eyelashes. Clin Exp Dermatol. 2010;35:e161-e162.
21. Smith JA. The impact of skin disease on the quality of life of adolescents. Adolesc Med. 2001;12(2):vii, 343-353. 


\section{Supplementary materials}

A

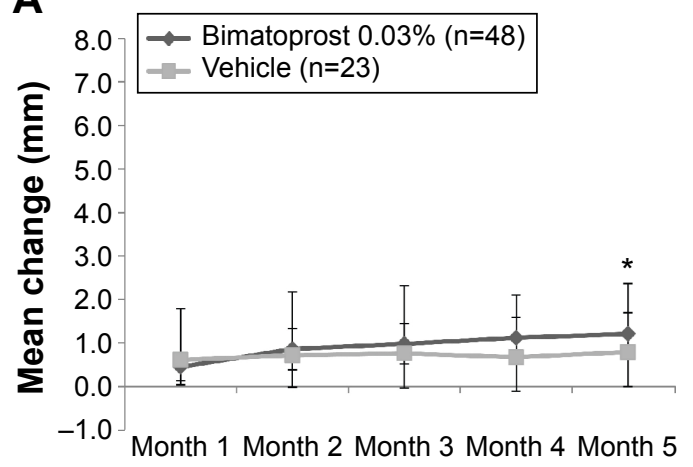

C $8.0 \rightarrow$ Bimatoprost $0.03 \%(n=13)$

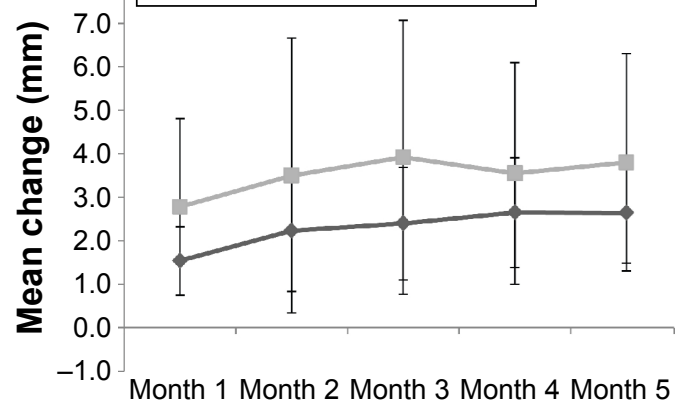

B

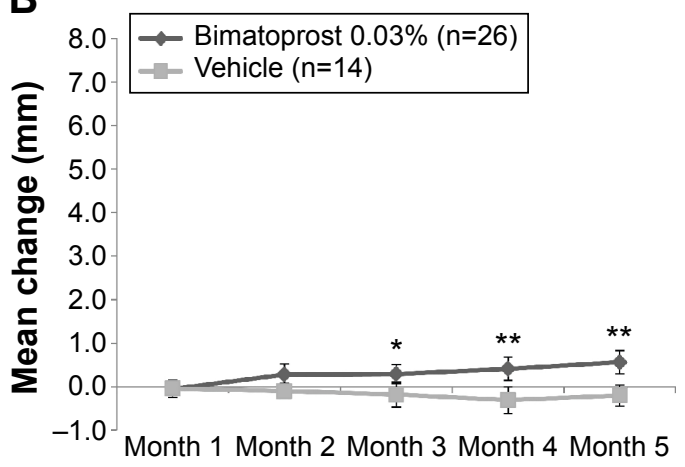

D $8.0 \rightarrow-$ Bimatoprost $0.03 \%(\mathrm{n}=9)$

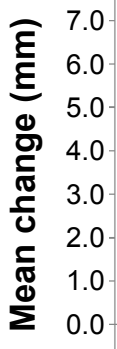

-1.0 Month 1 Month 2 Month 3 Month 4 Month 5

Figure SI Mean changes ( \pm 2 .SE) from baseline in upper eyelash length for (A) overall, (B) healthy adolescent, (C) postchemotherapy, and (D) alopecia areata pediatric populations.

Notes: Study treatment was applied once nightly for 4 months; subjects did not use study treatment between months 4 and 5 . $* P<0.02$ versus vehicle. $* * P<0.00 I$ versus vehicle.

A

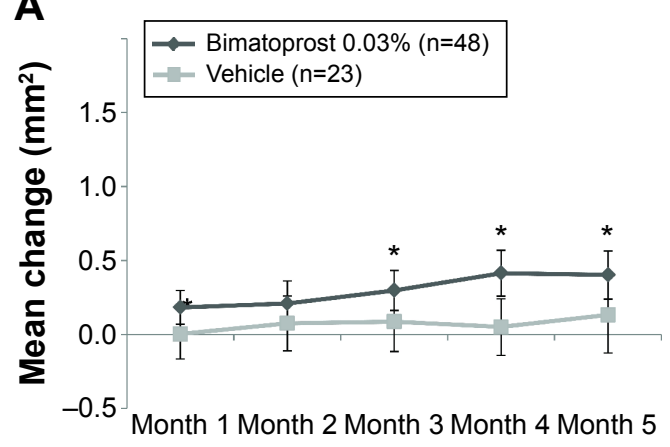

C

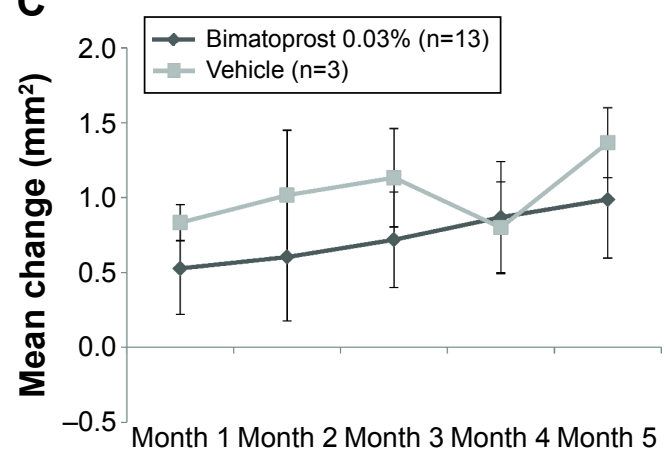

B

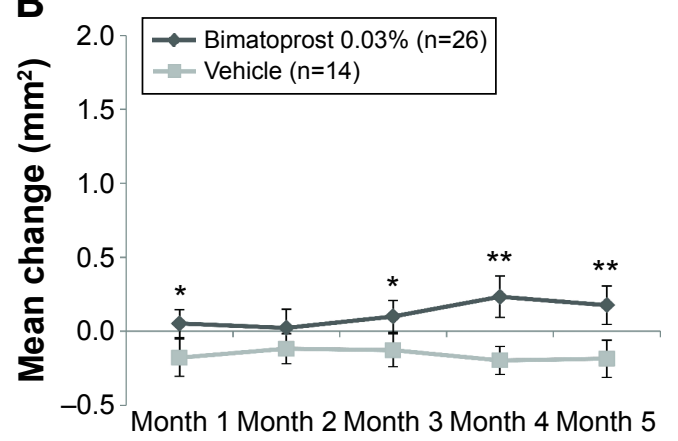

D

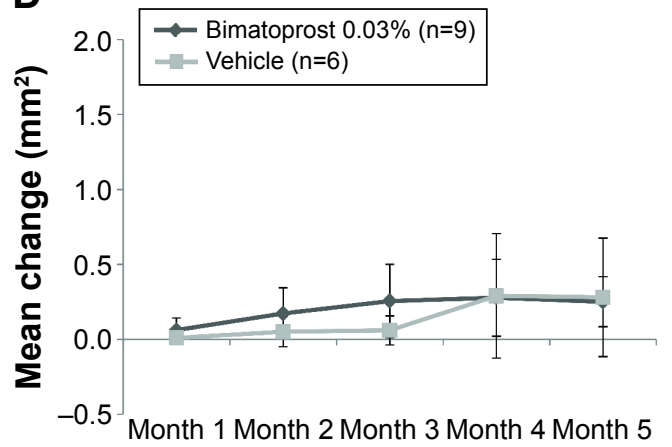

Figure S2 Mean changes $( \pm 2 \cdot S E)$ from baseline in upper eyelash thickness for $(\mathbf{A})$ overall, $(\mathbf{B})$ healthy adolescent, $(\mathbf{C})$ postchemotherapy, and (D) alopecia areata pediatric populations.

Notes: Study treatment was applied once nightly for 4 months; subjects did not use study treatment between months 4 and 5 . $* P<0.05$ versus vehicle. $* * P<0.00$ I versus vehicle. 

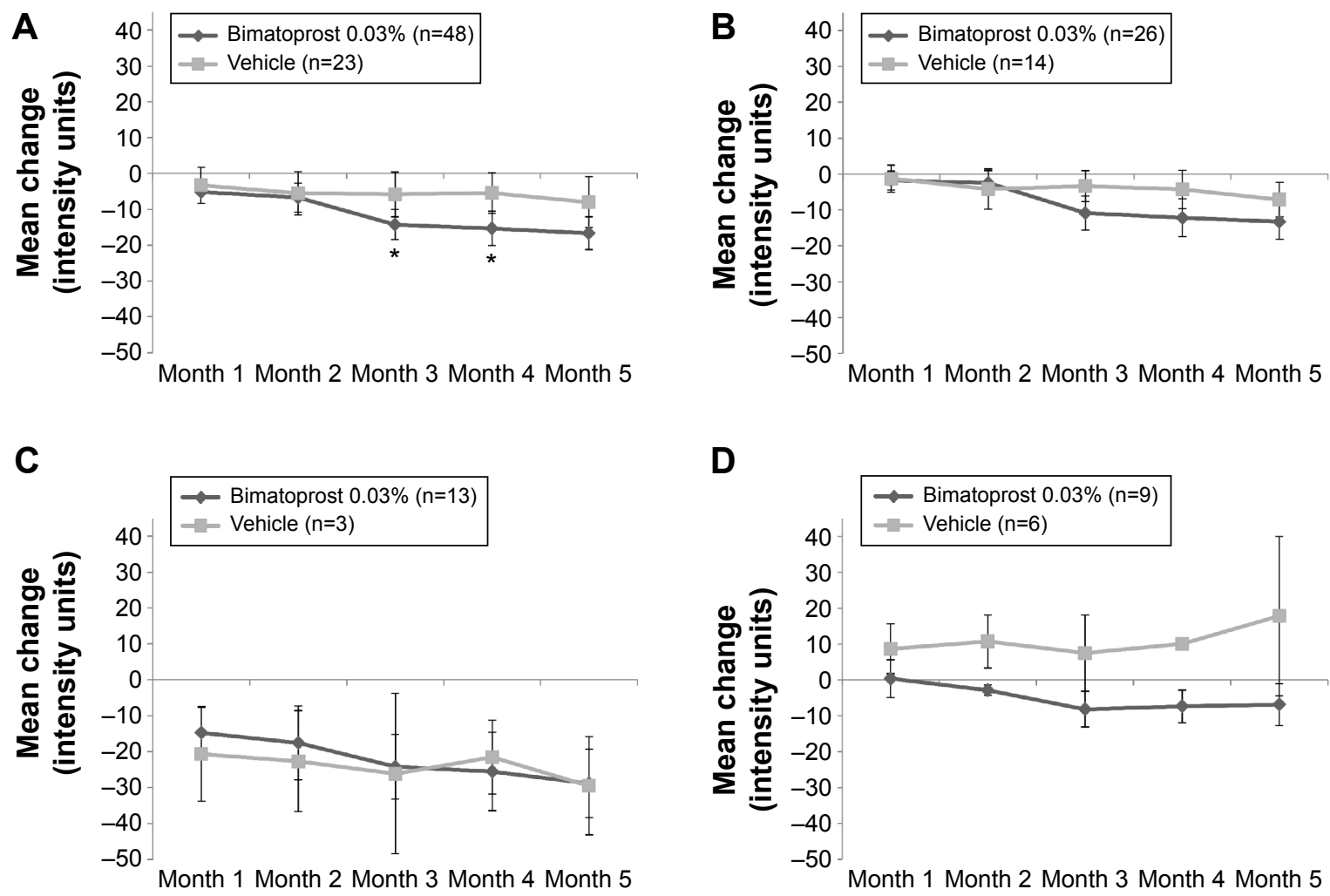

Figure S3 Mean changes ( \pm 2.SE) from baseline in upper eyelash darkness for (A) overall, (B) healthy adolescent, (C) postchemotherapy, and (D) alopecia areata pediatric populations.

Notes: Study treatment was applied once nightly for 4 months; subjects did not use study treatment between months 4 and 5 . Negative numbers indicate darker eyelashes compared with baseline. $* P<0.05$ versus vehicle.

\section{Publish your work in this journal}

Clinical Ophthalmology is an international, peer-reviewed journal covering all subspecialties within ophthalmology. Key topics include: Optometry; Visual science; Pharmacology and drug therapy in eye diseases; Basic Sciences; Primary and Secondary eye care; Patien Safety and Quality of Care Improvements. This journal is indexed on
PubMed Central and CAS, and is the official journal of The Society of Clinical Ophthalmology (SCO). The manuscript management system is completely online and includes a very quick and fair peer-review system, which is all easy to use. Visit http://www.dovepress.com/ testimonials.php to read real quotes from published authors. 